\title{
Receding Horizon-Based Dual Control Strategy for Pinpoint Planetary Landing
}

\author{
By Ping-Yuan CuI, ${ }^{1), 2)} \mathrm{Ai} \mathrm{GAO}^{1)}$ and $\mathrm{Hu}-\mathrm{Tao} \mathrm{CuI}{ }^{1)}$ \\ ${ }^{1)}$ Deep Space Exploration Research Center, Harbin Institute of Technology, Harbin, China \\ ${ }^{2)}$ Presently Key Laboratory of Dynamics and Control of Flight Vehicle, Ministry of Education, Beijing Institute of Technology, \\ Beijing, China
}

(Received August 22nd, 2011)

\begin{abstract}
A receding horizon-based dual control strategy for a planetary landing mission is developed. This strategy introduces the receding horizon framework to solve the nonlinear dynamic path planning problem with the state constraint, which makes up for the defects of the typical polynomial guidance law when it is used in landing on a planet with irregular gravity. Furthermore, the trade-off between efficient control and reliable estimation is considered. The cost incurred by the system uncertainty is incorporated into the performance index. Furthermore a linear feedback control law is provided with the quadratic performance index considering the dual features, which takes advantage of the nonlinear coupling between observability and trajectory to overcome the lack of observability and achieve better estimation performance. By stochastically optimizing the landing trajectory obtained from the receding horizon based convex programming method, the overall performance of the guidance, navigation and control (GNC) system for landing on planets is improved.
\end{abstract}

Key Words: Planetary Landing, Autonomous GNC Technology, Receding Horizon-Based Convex Programming, Feedback Dual Control

\section{Nomenclature}

$r$ : lander's position relative to landing site

$v$ : lander's velocity relative to landing site

$x$ : lander's state

$\hat{x}$ : estimated lander's state

$\tilde{x}$ : lander's state estimation error

$\mu$ : uncertainty state

$\chi$ : extended state

$a$ : acceleration

$g$ : gravity acceleration

$u$ : control input

$z$ : image processor output

$\omega$ : system process noise

$v$ : measurement noise

$f$ : camera's focal length

$t$ : time

$\Delta t$ : time interval

$T$ : desired maneuver time

$\kappa$ : constraint parameter

$\alpha$ : weighting coefficient of fuel

$\beta$ : weighting coefficient of energy

$\varepsilon$ : weighting coefficient of final state deviation

$W$ : weighting matrix

$\Pi$ : state constraint domain

$A$ : system matrix

$B$ : input matrix

$C$ : rotation matrix

$E$ : coefficient matrix

$Q$ : covariance matrix of system process noise
$R$ : covariance matrix of measurement noise

$P$ : error covariance matrix

$S$ : lower triangular matrix

$\Gamma$ : solution of algebraic Riccati equation

$K$ : feedback gain

$J$ : cost function

$\Psi$ : extended state vector function

$h$ : measurement output function

$\Sigma$ : coordinate system

Subscripts

ini: initial

fin: final

cur: current

des: desired

$s t d$ : steady state solution

cen: centrifugal

cor: Coriolis

unm: unmolded

$r$ : position

$v$ : velocity

$x$ : state

$\mu$ : uncertainty state

$\chi$ : extended state

$u$ : control

$k$ : discrete time step

$j$ : iteration number

$e$ : extended

$l$ : landing site

$c$ : camera

$i$ : inertial 


\section{Introduction}

The ultimate goal when landing on a planet is to reach the target landing point at the desired velocity, and thus the guidance and control law, which is crucial for successful landing operation, must be based on a best estimate of the system state. However, the planetary lander is subject to various sources of uncertainty during landing, such as gravitational uncertainty, environmental disturbance and measurement noise. Coupled with the long communication delay induced by the large distances between the target planet and ground stations on the earth, the traditional mode of navigating spacecraft with open loop control is not always suitable for pinpoint landing of a spacecraft on a planetary body. Instead, planetary landing operations must be performed autonomously using on-board sensors.

Typical control strategies are based on certain equivalence assumptions, namely that the choice of control actions does not affect nor is affected by the estimation uncertainty. This assumption allows to divide the guidance, navigation and control (GNC) system design to be divided into two separate processes: (i) estimate the system state from noise corrupted measurements using a navigation filter, and (ii) use classical polynomial trajectories ${ }^{1,2)}$ matched to the current state and target landing point to form way-points for lander tracking under the assumption that those estimated states are true.

However, solutions for dynamical systems are rarely simple polynomials, so tracking classical way-points is far from optimal. ${ }^{3-8)}$ Furthermore, for nonlinear systems, observability can also be affected by the system state trajectory due to a nonlinear coupling between observability and trajectory. ${ }^{9}$ ) When the observing capability is limited, achieving reliable landing performance becomes challenging, since this limited observing capability may lead to a lack of state observability. This may result in poor estimation performance and further on also weaken the performance of guidance and control measures. Therefore the question of how the overall performance of GNC can be optimized is an important problem to be solved in order to meet the future mission requirements for a low-cost and lightweight system.

In this study, monocular vision-based GNC for landing on planets is considered. A receding horizon-based dual control strategy is suggested to solve the problem of typical guidance and control strategies. This strategy introduces a receding horizon framework ${ }^{10-14)}$ to solve the convex optimization problem that incorporates the gravity models of target celestial bodies with the development of the way-points. On this basis, it incorporates the cost incurred by the system uncertainty into the performance index to provide a linear feedback control law with the quadratic performance index considering the dual features, which take advantage of the nonlinear coupling between observability and trajectory to overcome the lack of observability and to achieve better estimation performance. By stochastically optimizing the landing trajectory obtained from the receding horizon-based convex programming method, the overall performance of GNC for landing on planets is improved.

This paper is structured as follows. Section 2 describes the system dynamics for planetary landing and formulates the monocular vision-based GNC problem. The receding horizon-based convex optimization algorithm is developed in section 3. The feedback dual control is derived in section 4. Section 5 presents the simulation results and section 6 contains the concluding remarks.

\section{System Dynamics Model and Problem Formulation}

First, the landing site coordinate system $\Sigma^{l}: o_{l}-x_{l} y_{l} z_{l}$ is defined in order to set up the lander dynamics model. Let the landing site coordinate system be fixed on the planet with the origin coinciding with the landing site $\left(o_{l}\right)$, and the $x_{l^{-}}$ axis coinciding with the unit vector from the mass center of the planet to the landing site. The $z_{l}$-axis points to the south direction along the meridian tangent direction. The $x_{l}, y_{l}$ and $z_{l}$ axes compose a right-handed coordinate system (see Fig. 1).

The coordinate system $\Sigma^{i}: o_{i}-x_{i} y_{i} z_{i}$ is the planetocentric inertial coordinate system with the origin coinciding with the planet mass center $\left(o_{i}\right)$, the $z_{i}$-axis coinciding with the planet's maximum inertia axis, and the $x_{i}$-axis coinciding with the minimum inertia axis at epoch time. The definition of the $y_{i}$-axis satisfies the right-hand rule.

Then, the equations of motion are represented in the landing coordinate system as follows

$$
\begin{aligned}
& \dot{r}=\dot{v} \\
& \dot{v}=u+g-a_{c e n}-a_{c o r}+a_{\text {unm }}
\end{aligned}
$$

Processing the acceleration term $-a_{c e n}-a_{c o r}+a_{\text {unm }}$ in Eq. (1) as the system process noise, the dynamic model is written as follows:

$$
\begin{aligned}
\dot{x}(t) & =\left[\begin{array}{ll}
0 & I \\
0 & 0
\end{array}\right] x(t)+\left[\begin{array}{l}
0 \\
I
\end{array}\right]\left\{u(t)+g\left(E_{r} x(t)\right)+\omega(t)\right\} \\
& =A x(t)+B\left\{u(t)+g\left(E_{r} x(t)\right)+\omega(t)\right\}
\end{aligned}
$$

where,

$$
\begin{aligned}
& \left.x=\left[\begin{array}{ll}
r & v
\end{array}\right]^{\mathrm{T}}, \quad A=\left[\begin{array}{ll}
0 & 0
\end{array}\right]^{\mathrm{T}} \quad\left[\begin{array}{ll}
I & 0
\end{array}\right]^{\mathrm{T}}\right] \text {, } \\
& B=\left[\begin{array}{ll}
0 & I
\end{array}\right]^{\mathrm{T}}, \quad E_{r}=\left[\begin{array}{ll}
I & 0
\end{array}\right],
\end{aligned}
$$

and $\omega$ is a zero mean Gaussian noise with covariance matrix $Q$.

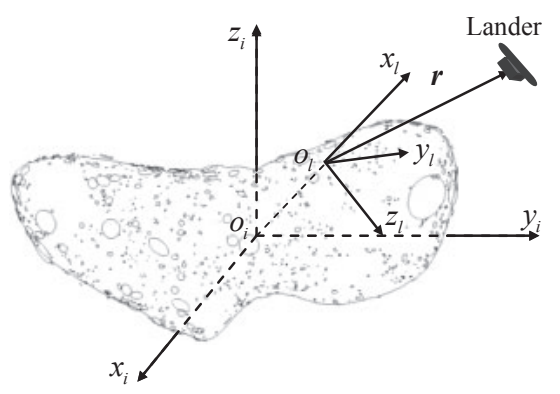

Fig. 1. Definition of coordinate system. 
Considering monocular vision-based navigation, let $C_{c l}$ denote a known camera attitude represented by a rotation matrix from the landing site coordinate system to the camera coordinate system. Then the image processor output at the $k$-th time step is modeled as

$$
\begin{aligned}
z_{k} & =h\left(x_{c}\left(t_{k}\right)\right)+v_{k} \\
& =\left[\begin{array}{ll}
f \frac{r_{c y}}{r_{c x}} & f \frac{r_{c z}}{r_{c x}}
\end{array}\right]^{\mathrm{T}}+v_{k}
\end{aligned}
$$

where $v_{k}$ is a zero mean Gaussian noise with covariance $R_{k}$, and

$$
r_{c}(t)=C_{c l} r(t)=\left[\begin{array}{lll}
r_{c x} & r_{c y} & r_{c z}
\end{array}\right]^{\mathrm{T}} .
$$

Now the problem of monocular vision-based GNC of planetary landing can be described as follows. The lander's current state is estimated using the optical observation information described in Eq. (3) and the estimated state is used for the guidance law, which plans for the lander to reach the target landing site from the current location. Then the guidance given to the lander can be represented as the following quadratic minimization problem:

$$
\begin{aligned}
\min _{u(t)} J= & \left\{x\left(t_{\text {fin }}\right)-x_{\text {fin }}\right\}^{\mathrm{T}} W_{x}\left\{x\left(t_{\text {fin }}\right)-x_{\text {fin }}\right\} \\
& +\int_{t_{0}}^{t_{\text {fin }}} u^{\mathrm{T}}(t) W_{u} u(t) \mathrm{d} t
\end{aligned}
$$

where,

$$
W_{x} \geq 0, \quad W_{u}>0
$$

\section{Receding Horizon-Based Feed-Forward Guidance Algorithm}

Considering a single thruster control cycle $\Delta t$, the system described by Eq. (2) is discretized on each time interval $\Delta t=t_{k+1}-t_{k}$ as follows:

$$
x_{k+1}=A_{k} x_{k}+B_{k} u_{k}+B_{k} g_{k}
$$

where,

$$
A_{k}=e^{A \Delta t}, \quad B_{k}=\int_{0}^{\Delta t} e^{A(\Delta t-\tau)} B \mathrm{~d} \tau,
$$

and $g_{k}$ are the numerical gravity gradients.

Given a trajectory maneuver requirement with a desired maneuver time $T$, an initial state $x_{i n i}$, and a desired final state $x_{f i n}$, an initial reference trajectory based on a polynomial fit is set such that $x_{0}^{0}=x_{i n i}$ (i.e., $t_{0}=0$ ) and $x_{N}^{0}=x_{f i n}$ (i.e., $\left.t_{N}=T\right)$. Simultaneously, the $g_{k}^{0}$ value is determined for each $x_{k}^{0}$. Beginning with the initial reference trajectory, the framework of receding horizon is introduced to solve the dynamic trajectory programming problem. The current state $x_{c u r}$ (i.e., the estimated state obtained from the navigation system) is used as the initial state and the desired state constraint is imposed upon a desired cost function as Eq. (6). Then an update to $x_{k}$, along with values for $u_{k}$, is determined. The solution $\left\{x_{k=j-1}^{j}, \cdots, x_{k=N}^{j}\right\}(j>0)$ is used to determine the gravity $\left\{g_{k=j}^{j}, \cdots, g_{k=N-1}^{j}\right\}(j>0)$ and then fed back

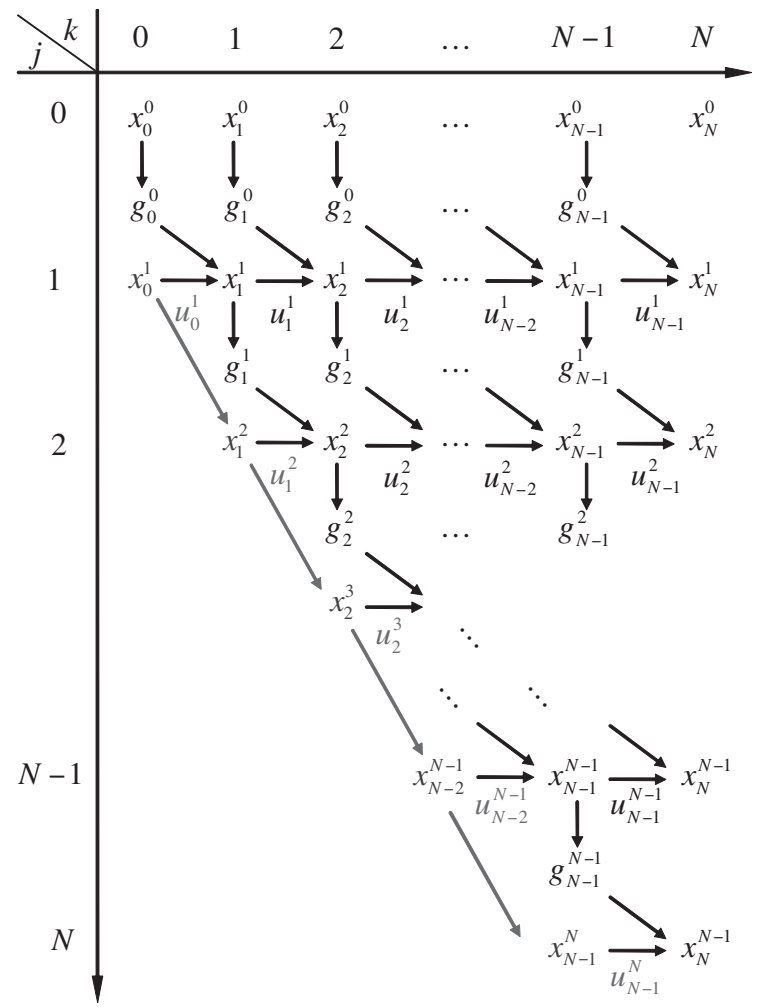

Fig. 2. Schematic diagram of the solution process.

through the discrete dynamics in Eq. (5). In the optimal control sequence $\left\{u_{k=j-1}^{j}, \cdots, u_{k=N-1}^{j}\right\}(j>0)$, only the first control $u_{k=j-1}^{j}$ is applied to the current system. This iterative procedure is used to solve the feed-forward guidance problem and provide an admissible solution $\left\{x_{k}, u_{k}\right\} \quad(k=$ $0, \cdots, N-1$ ) to system (5) (see Fig. 2).

Minimize

$$
\begin{aligned}
& \sum_{k=j-1}^{N-2}\left(\alpha\left\|u_{k+1}^{j+1}\right\|+\beta\left\|u_{k+1}^{j+1}\right\|^{2}\right) \\
& \quad+\varepsilon\left\|E_{v}\left(x_{N}^{j+1}-x_{\text {fin }}\right)\right\|
\end{aligned}
$$

subject to

$$
\begin{aligned}
& x_{k+2}^{j+1}=A_{k+1} x_{k+1}^{j+1}+B_{k+1} u_{k+1}^{j+1}+B_{k+1} g_{k+1}^{j} \\
& (k=j-1, \cdots, N-2) \\
& x_{k+1}^{j+1}=x_{\text {cur }} \quad(k=j-1) \\
& E_{r}\left(x_{N}^{j+1}-x_{\text {fin }}\right)=0 \\
& x_{k+1}^{j+1} \in \Pi \quad(k=j-1, \cdots, N-2) \\
& \left\|x_{k+1}^{j+1}-x_{k+1}^{j}\right\| \leq \kappa\left\|x_{k+1}^{j}-x_{k+1}^{j-1}\right\| \\
& (k=j-1, \cdots, N-2)
\end{aligned}
$$

Here $(\alpha, \beta)=(1,0)$ is used for the minimum fuel problem and $(\alpha, \beta)=(0,1)$ is used for the energy problem. The final state constraints are relaxed. $E_{r}=\left[\begin{array}{ll}I & 0\end{array}\right]$ is for the equality constraint at the final state, $E_{v}=\left[\begin{array}{ll}0 & I\end{array}\right]$ is for the contribution of the final state to the cost, and $\varepsilon>0$. $\Pi$ is a convex set. The given constraint parameter $0<\kappa<1$ assures the algorithm generates a convergent set of trajectories. 


\section{Stochastic Feedback Dual Control Algorithm}

From the above, the guidance design is based on the estimated state obtained from the navigation system. Therefore, the quality of positioning affects the performance of the guidance and control system, and this is the prerequisite for ensuring a successful planetary landing.

For linear systems, observability depends only on the system dynamics and the sensor model. However, for nonlinear systems, observability can also be affected by the system state trajectory due to the nonlinear coupling between observability and trajectory. In fact, if the system is dynamically observable, this coupling can be exploited to overcome the lack of observability and thus achieve better estimation performance.

In particular, for monocular vision-based landing, the system's observability can be greatly improved by performing some motions. However, there exist an infinite number of feasible trajectories, and calculating an optimal trajectory is a challenging stochastic optimization task. ${ }^{15,16)}$ The use of standard certainty equivalence control techniques and various other approximation techniques is limited due to the inherent complexity of the problem, including the trade-off between efficient control and reliable estimation. ${ }^{17-21)}$ In this paper, a dual control algorithm considering such a trade-off is derived. To improve estimation accuracy, it incorporates the cost incurred by the system uncertainty into the performance index. In addition, a linear feedback control law is provided to optimize the receding horizonbased feed-forward guidance trajectory while applying a linear quadratic control technique.

Considering the lander state $x$ to be represented as a cloud of particles $x_{i}(i=1,2, \cdots, N)$, which are distributed in phase space, the cost can be calculated as the sequential sum of squared distances between the desired state $x_{\text {des }}$ and each particle $x_{i}$. The cost reflects both the distance between those particles, the desired state and how severely scattered they are. The quality of control at a particular time can be expressed using the following quadratic expression.

$$
\begin{aligned}
\delta J & =\frac{1}{N} \sum_{i=1}^{N}\left\{\left(x_{i}-x_{d e s}\right)^{\mathrm{T}} W_{x}\left(x_{i}-x_{d e s}\right)+u^{\mathrm{T}} W_{u} u\right\} \\
& =E\left[\left(x-x_{d e s}\right)^{\mathrm{T}} W_{x}\left(x-x_{d e s}\right)+u^{\mathrm{T}} W_{u} u\right]
\end{aligned}
$$

Define $\hat{x}$ as the estimated state $x$ of the lander. Then the estimation error is $\tilde{x}=x-\hat{x}$, which satisfies $E[\tilde{x}]=0$, $P=E\left[\tilde{x}^{\mathrm{T}} \tilde{x}\right]$. Then Eq. (7) can be rewritten as

$$
\begin{aligned}
\delta J & =E\left[\left(\hat{x}+\tilde{x}-x_{d e s}\right)^{\mathrm{T}} W_{x}\left(\hat{x}+\tilde{x}-x_{d e s}\right)+u^{\mathrm{T}} W_{u} u\right] \\
& =\left(\hat{x}-x_{d e s}\right)^{\mathrm{T}} W_{x}\left(\hat{x}-x_{d e s}\right)+u^{\mathrm{T}} W_{u} u+E\left[\tilde{x}^{\mathrm{T}} W_{x} \tilde{x}\right] \\
& =\left(\hat{x}-x_{d e s}\right)^{\mathrm{T}} W_{x}\left(\hat{x}-x_{d e s}\right)+u^{\mathrm{T}} W_{u} u+\operatorname{tr}\left[W_{x} P\right]
\end{aligned}
$$

The first two terms in Eq. (8) are in a familiar quadratic form. Expressing the third term in a quadratic form enables solutions that exploit existing mathematical tools. This can be done by noting that the error covariance $P$ is a positive semi-definite matrix, thus there exists an unique lower triangular matrix $S \in R^{6 \times 6}$ that satisfies $P=S S^{\mathrm{T}}$, i.e., the square root matrix of $P$ obtained by lower triangular decomposition is as follows.

$$
S=\left[\begin{array}{cccc}
s_{11} & 0 & \cdots & 0 \\
s_{21} & s_{22} & \cdots & 0 \\
\vdots & \vdots & \ddots & \vdots \\
s_{61} & s_{62} & \cdots & s_{66}
\end{array}\right]
$$

For convenience, assume that $W_{x}$ is a diagonal matrix and $W_{x}(i, i)=w_{i i}(i=1,2, \cdots, 6)$. Then the last term in Eq. (8) is expressed as

$$
\operatorname{tr}\left[W_{x} P\right]=\operatorname{tr}\left[W_{x} S^{\mathrm{T}} S\right]=\sum_{i=1}^{6}\left\{w_{i i} \sum_{j=1}^{i} s_{i j}^{2}\right\}
$$

Hence, Eq. (10) can be rewritten as

$$
\begin{aligned}
\delta J= & \left(\hat{x}-x_{d e s}\right)^{\mathrm{T}} W_{x}\left(\hat{x}-x_{d e s}\right)+u^{\mathrm{T}} W_{u} u \\
& +\sum_{i=1}^{n}\left\{w_{i i} \sum_{j=1}^{i} s_{i j}^{2}\right\}
\end{aligned}
$$

This is the quadratic function of the estimated state deviation, the control input, and the nonzero elements of the square root of the error covariance matrix.

Integrating Eq. (11) over time yields the performance index to be minimized for stochastic optimization.

$$
\begin{aligned}
J= & \int_{0}^{t_{f i n}}\left\{\left(\hat{x}-x_{d e s}\right)^{\mathrm{T}} W_{x}\left(\hat{x}-x_{d e s}\right)+u^{\mathrm{T}} W_{u} u\right. \\
& \left.+\sum_{i=1}^{6}\left\{w_{i i} \sum_{j=1}^{i} s_{i j}^{2}\right\}\right\} \mathrm{d} t
\end{aligned}
$$

The first two terms of Eq. (12) correspond to the certainty equivalence control cost, and the third term corresponds to the cost due to uncertainties in the system.

Define the expanded state $\chi=\left[\begin{array}{ll}x & \mu\end{array}\right]_{x=\hat{x}}^{\mathrm{T}}$, and the uncertainty state vector $\mu=\left[\begin{array}{lllll}s_{11} & s_{21} & s_{22} & \cdots & s_{66}\end{array}\right]^{\mathrm{T}}$ containing all the nonzero elements in $S$. For descriptive convenience, the desired state $x_{d e s}$ is set to zero hereafter without loss of generality. Then, the quadratic performance index in Eq. (12) becomes

$$
J=\int_{0}^{t_{\text {fin }}}\left\{\chi^{\mathrm{T}} W_{e} \chi+u^{\mathrm{T}} W_{u} u\right\} \mathrm{d} t
$$

where,

$$
\begin{aligned}
W_{e}= & {\left[\begin{array}{cc}
W_{x} & 0 \\
0 & W_{\mu}
\end{array}\right], } \\
W_{\mu}= & {\left[\begin{array}{ccccc}
w_{11} & 0 & 0 & \cdots & 0 \\
0 & w_{22} & 0 & \cdots & 0 \\
0 & 0 & w_{22} & \cdots & 0 \\
\vdots & \vdots & \vdots & \ddots & \vdots \\
0 & 0 & 0 & 0 & w_{66}
\end{array}\right]_{21 \times 21} }
\end{aligned}
$$

Hereto, the optimization problem can be described as follows.

Find the optimal control input $u^{*}(t)$ in the time interval $\left(0, t_{\text {fin }}\right)$ that satisfies 


$$
u^{*}=\arg \min _{u} \int_{0}^{t_{\text {fin }}}\left\{\chi^{\mathrm{T}} W_{e} \chi+u^{\mathrm{T}} W_{u} u\right\} \mathrm{d} t
$$

Subject to the extended phase space description

$$
\dot{\chi}=\left[\begin{array}{ll}
\dot{x} & \dot{\mu}
\end{array}\right]^{\mathrm{T}}=\Psi(\chi, u)
$$

As a result of the approximation involved in the covariance propagation step and the complexity of its state space representation, it can be a difficult task to calculate the optimal solution for this nonlinear control problem. In this study, the suboptimal solution of the control problem is obtained by state-feedback control using an infinite horizon steady-state gain.

Assume that the Riccati differential equation for the preceding optimization problem has a limiting solution $\Gamma$, which satisfies the algebraic Riccati equation

$$
0=A_{e}^{\mathrm{T}} \Gamma+\Gamma A_{e}+W_{e}-\Gamma B_{e} W_{u}^{-1} B_{e}^{\mathrm{T}} \Gamma
$$

where,

$$
A_{e}=\left.\frac{\partial \Psi(\chi, u)}{\partial \chi}\right|_{x=\hat{x}}, \quad B_{e}=\left.\frac{\partial \Psi(\chi, u)}{\partial u}\right|_{x=\hat{x}}
$$

then, the state-feedback control law is as follows:

$u=-\left.K\left(\chi-\chi_{s t d}\right)\right|_{x=\hat{x}}=-K_{x}\left(\hat{x}-x_{d e s}\right)-K_{\mu}\left(\mu-\mu_{s t d}\right)$

where

$$
K=W_{u}^{-1} B_{e}^{\mathrm{T}} \Gamma=\left[\begin{array}{ll}
K_{x} & K_{\mu}
\end{array}\right] .
$$

$K_{x}$ focuses primarily on the control goal of stabilization, and $K_{\mu}$ provides an additional feedback input driven by the uncertainty state, which decreases uncertainties of the control system in a direct manner and improves the control performance indirectly.

\section{Simulation Results}

In order to confirm the validity of the receding horizonbased dual control strategy proposed in this paper, it is tested using asteroid landing scenarios, and simulations are performed in a matlab environment.

The asteroid gravity environment used in the simulations is based on Eros data, which contain up to fourth-order spherical harmonics.

In the landing coordinate system the lander's initial state is $x_{i n i}=\left[\begin{array}{llllll}500 & 20 & -50 & 1.5 & 2 & 0\end{array}\right]^{\mathrm{T}} \mathrm{m}$ and the desired maneuver time is $T=300 \mathrm{~s}$.

When perfect estimation is achieved under no uncertainty, as shown in Fig. 3, the lander can be sent to the target landing point with an error range of $2 \mathrm{~m}$, and the final landing velocity can be controlled within approximately $0.02 \mathrm{~m} / \mathrm{s}$. Here, only the control input obtained by the receding horizon-based feed-forward guidance algorithm is used.

However, when considering estimation error of the lander state, where the initial position and velocity estimation errors are subject to random distribution of $100 \mathrm{~m}^{2}$ and $0.1 \mathrm{~m}^{2} / \mathrm{s}^{2}$, respectively, as shown in Fig. 4, the lander

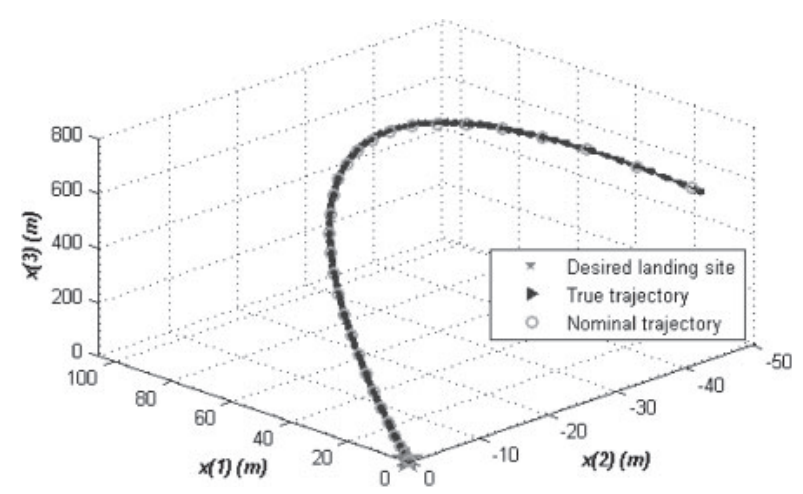

Fig. 3. Landing trajectory under no uncertainty.

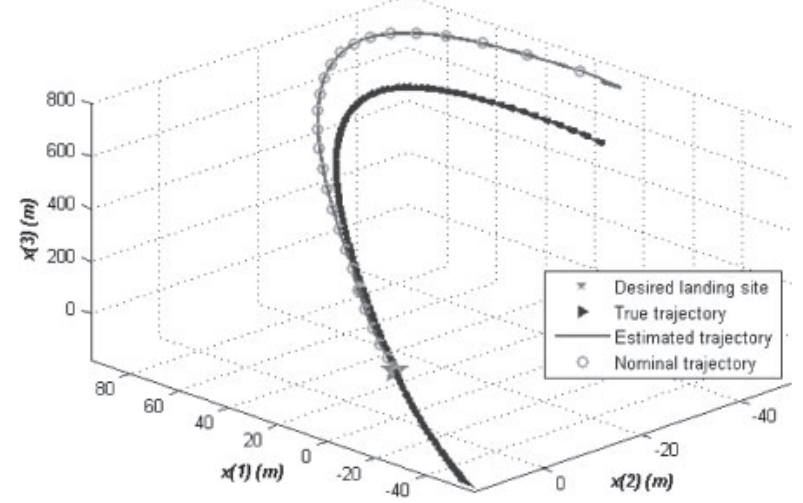

Fig. 4. Landing trajectory under the influence of navigation error.

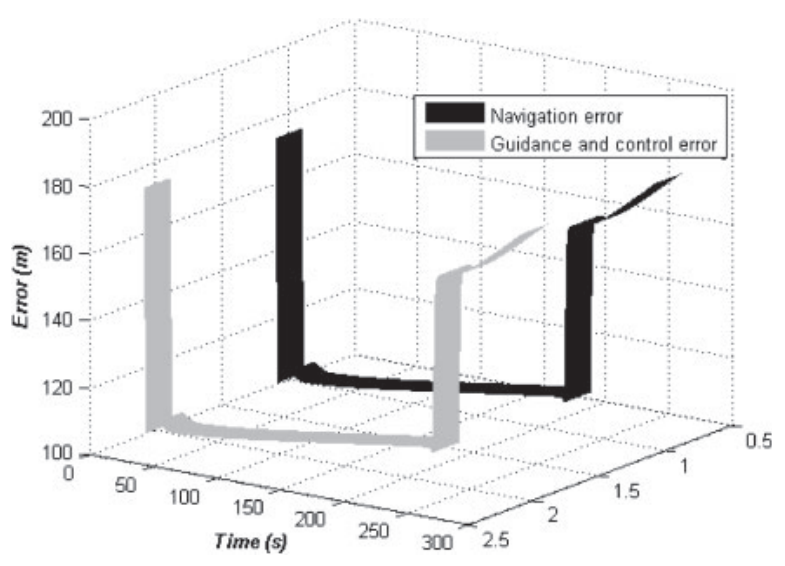

Fig. 5. Comparison of navigation and guidance performance.

deviates from the target landing site and the final landing velocity is up to $3 \mathrm{~m} / \mathrm{s}$. The result is that the lander crashes into the target body within the expected landing time. This is mainly because the observability of the lander state is affected by the landing trajectories. As shown in Fig. 5, when the observing capability is limited, the estimation error will increase, and reduce the guidance and control performance.

The addition of feedback dual control input significantly improves landing precision. Figure 6 shows that the estimation error of the lander's state decreases along the landing trajectory, and in the final phase, the landing state is almost 


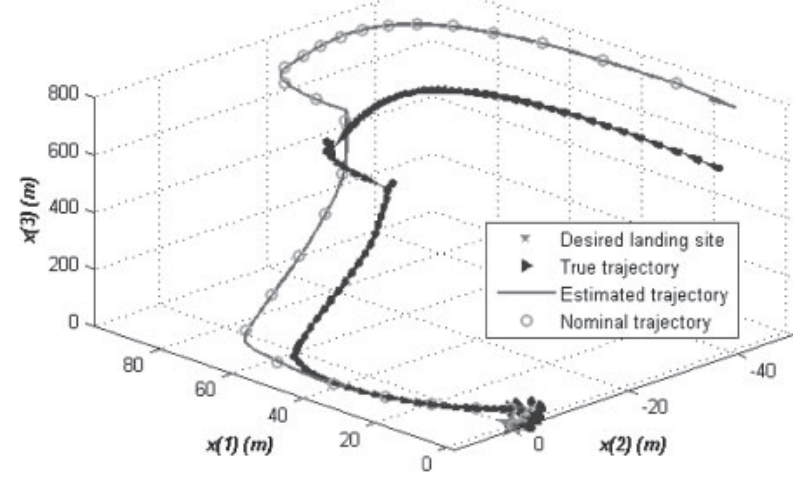

Fig. 6. Landing trajectory by stochastic optimization.

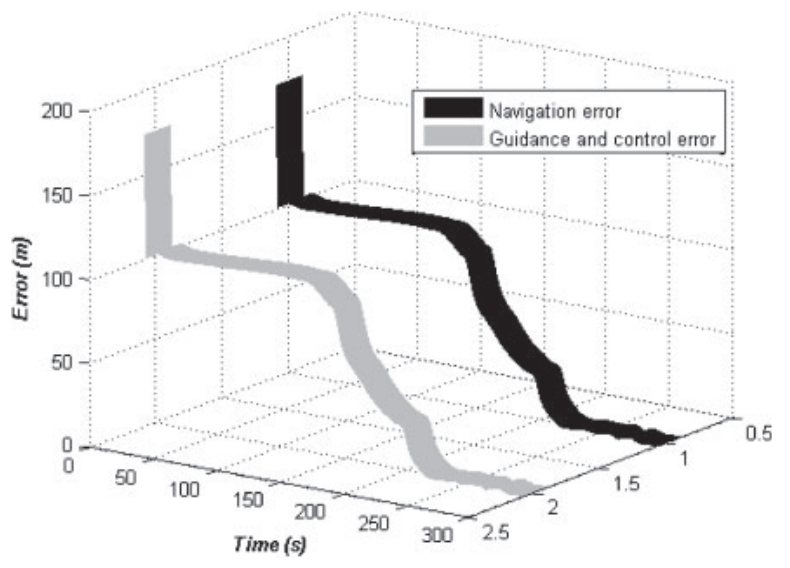

Fig. 7. Comparison of navigation and guidance performance.

coincident with the way-point generated by the receding horizon-based feed-forward guidance algorithm. The final landing position deviation is $5 \mathrm{~m}$ and the landing velocity is about $0.05 \mathrm{~m} / \mathrm{s}$. As shown in Fig. 7, the overall performance of the GNC system is improved when the feedback dual control input is added to optimize the trajectory calculated by the receding horizon-based feed-forward guidance algorithm.

Considering the relation between the navigation accuracy and the horizontal motion of the lander, as shown in Figs. 8 and 9 , the horizontal motion planning given by the receding horizon-based feed-forward guidance algorithm is smooth. Particularly during the terminal landing phase, there is little change in the horizontal motion and the navigation accuracy decreases continuously, while the magnitude of the lander's horizontal motion increases by adding the feedback dual control input. Therefore, the estimation error decreases rapidly, and the navigation accuracy increases. It is thus clear that the feedback dual control input optimizes the trajectory given by the receding horizon-based feed-forward guidance algorithm by adding the magnitude of the lander's horizontal motion, which leads to improved observability of the system. Further, comparing the control cost with the total cost as shown in Fig. 10, even though the control cost increases due to the addition of feedback dual control input, the total cost is significantly reduced.

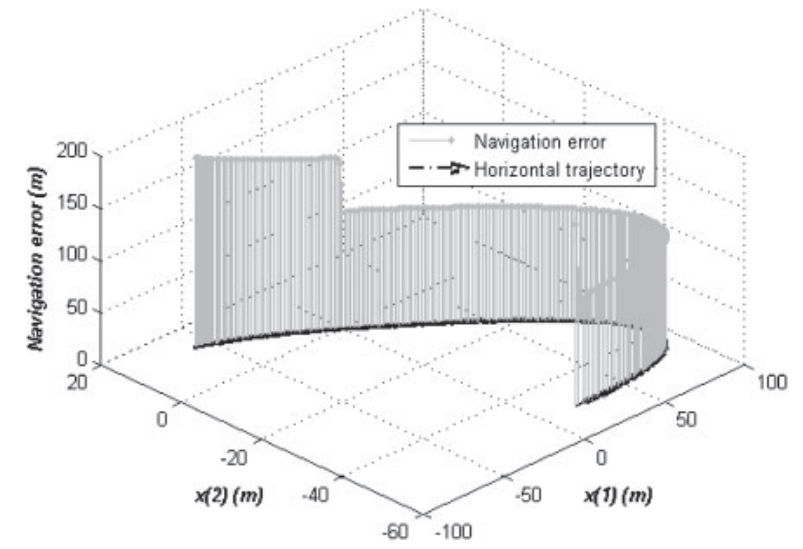

Fig. 8. Change trend of the horizontal motion effecting navigation accuracy.

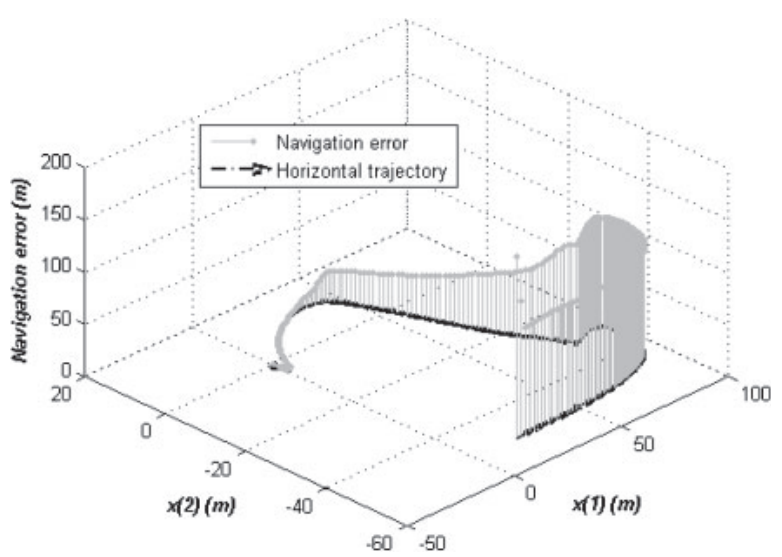

Fig. 9. Change trend of the horizontal motion effecting navigation accuracy by stochastic optimization.
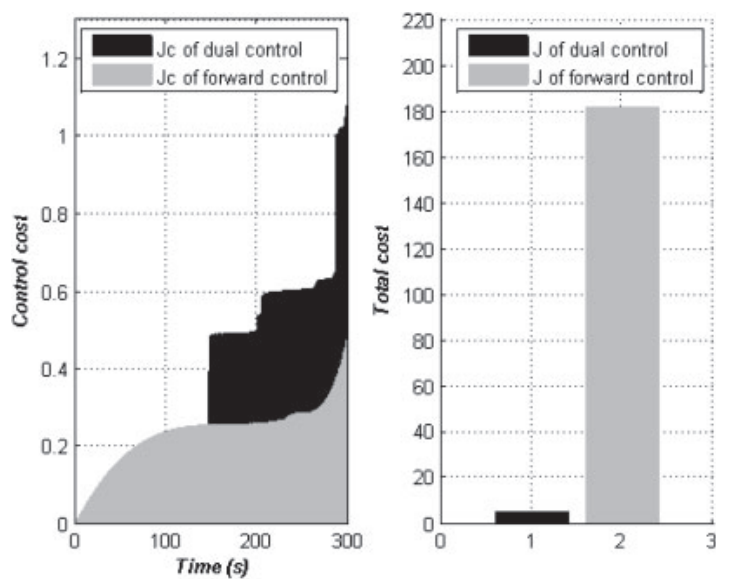

Fig. 10. Comparison of control cost and total cost.

\section{Conclusion}

This paper proposed a receding horizon-based dual control strategy for pinpoint planetary landing. A nominal trajectory with state and nonlinear dynamic constraints was generated by solving the convex optimization problem that incorporates the gravity models of the target celestial body under a receding horizon framework. Subsequently, 
the nominal landing trajectory was optimized by minimizing the performance index in a quadratic form with the system uncertainty extended into the system state. This procedure takes advantage of the nonlinear coupling between observability and trajectory in order to overcome the lack of observability and to achieve better estimation performance. The simulation analysis shows that the receding horizon-based dual control strategy improves the navigation accuracy, along with meeting the requirements of a planetary landing mission, which leads to significant improvement of the overall system performance by stochastically optimizing the nominal trajectory at the price of control cost.

\section{Acknowledgments}

The research described in this paper is supported by the National Basic Research Program of China (973 Program) (2012CB720000), the National Natural Science Foundation of China (60874094), the State Key Program of National Natural Science of China (10832004), the National High Technology Research and Development Program of China (2010AA122206) and the Defense Industrial Technology Development Program of China (B2220110012). The authors wish to thank Norbert Koemle from the Space Research Institute, Austrian Academy of Sciences for helpful suggestions to improve this paper.

\section{References}

1) Klumpp, A. R.: Apollo Lunar Descent Guidance, Automatica, 10 (1974), pp. 133-146.

2) Sostaric, R. R. and Rea, J. R.: Powered Descent Guidance Methods for the Moon and Mars, American Institute of Aeronautics and Astronautics Inc., San Francisco, 2005.

3) Acikmese, A. B. and Carson, J. M.: Small Body GN\&C Research Report: A Robust Model Predictive Control Algorithm, Internal Document JPL D-32947, Jet Propulsion Laboratory, California Institute of Technology, Pasadena, 2005.

4) Acikmese, A. B. and Carson, J. M.: A Nonlinear Model Predictive Control Algorithm with Proven Robustness and Resolvability, Institute of Electrical and Electronics Engineers Inc., New Jersey, 2006.
5) Blackmore, L., Acikmese, B. and Scharf, D.: Minimum-landingerror Powered-descent Guidance for Mars Landing Using Convex Optimization, J. Guid. Control Dynam., 33 (2010), pp. 1161-1171.

6) Acikmese, A. B. and Ploen, S. R.: A Powered Descent Guidance Algorithm for Mars Pinpoint Landing, American Institute of Aeronautics and Astronautics Inc., Reston, VA, 2005.

7) Ploen, S. R., Acikmese, A. B. and Wolf, A.: A Comparison of Powered Descent Guidance Laws for Mars Pinpoint Landing, American Institute of Aeronautics and Astronautics Inc., Reston, VA, 2006.

8) Acikmese, A. B. and Ploen, S. R.: Convex Programming Approach to Powered Descent Guidance for Mars Landing, J. Guid. Control Dynam., 30 (2007), pp. 1353-1366.

9) Metthies, L., Kanade, T. and Szeliski, R.: Kalman Filter-based Algorithms for Estimating Depth from Image Sequences, Int. J. Comput. Vision, 3 (1989), pp. 209-238.

10) Mayne, D. Q., Rawlings, J. B., Rao, C. V. and Scokaert, P. O. M.: Constrained Model Predictive Control: Stability and Optimality, Automatica, 36 (2000), pp. 789-814.

11) Magni, M., Nijmeijer, H. and Schaft van der, A. J.: A Receding Horizon Approach to the Nonlinear $H_{\infty}$ Control Problem, Automatica, 37 (2001), pp. 429-435.

12) Bemporad, A., Morari, M., Dua, V. and Pistikopoulos, E. N.: The Explicit Linear Quadratic Regulator for Constrained Systems, Automatica, 38 (2002), pp. 3-20.

13) Bemporad, A., Borelli, F. and Morari, M.: Model Predictive Control Based on Linear Programming-The Explicit Solution, IEEE Trans. Autom. Control, 47 (2002), pp. 1974-1985.

14) Smith, R. S.: Robust Model Predictive Control of Constrained Linear Systems, Proceedings of the American Control Conference, 2004.

15) Kirk, D. E.: Optimal Control Theory, Prentice-Hall, Englewood Cliffs, NJ, 1970.

16) Bryson, A. E. and Ho, Y. C.: Applied Optimal Control, Hemisphere, New York, 1975, pp. 131-141.

17) Feldbaum, A. A.: Dual Control Theory I, Automation Remote Control, 21 (1960), pp. 874-880.

18) Feldbaum, A. A.: Dual Control Theory II, Automation Remote Control, 21 (1960), pp. 1033-1039.

19) Feldbaum, A. A.: Dual Control Theory III, Automation Remote Control, 22 (1961), pp. 1-12.

20) Feldbaum, A. A.: Dual Control Theory IV, Automation Remote Control, 22 (1961), pp. 109-121.

21) Tse, E., Bar-Shalom, Y. and Meier, L.: Wide-Sense Adaptive Dual Control for Nonlinear Stochastic Systems, IEEE Trans. Autom. Control, 18 (1973), pp. 98-108. 